\title{
Detection of Jumps Parameters in Economic Processes(the Case of Modelling Profitability)
}

\author{
O.V. Poliarus ${ }^{1 *}$, Y.O. Poliakov ${ }^{1}$, I. L. Nazarenko ${ }^{2}$, Y. T. Borovyk ${ }^{2}$, M. V. Kondratiuk ${ }^{2}$ \\ ${ }^{1}$ Kharkiv National Automobile and Highway University \\ ${ }^{2}$ Ukrainian State University of Railway Transport \\ *Corresponding author E-mail: poliarus.kharkiv@gmail.com
}

\begin{abstract}
A new method of parameters jumps detection in economic processes is presented. A jump of the economic process parameter must be understood as a rapid parameter change for a time that does not exceed the period of process registration. A system of stochastic differential equations for a posteriori density probability of a jump is synthesized. The solution of the system is the probability of a parameter jump, the estimation and variance of the jump in the presence of a priori information under conditions of noise influence. The simulation results are conducted for profitability of machine building industry of Kharkiv region, Ukraine. The system provides detection of jump parameters, even in conditions of intense noise of economic nature. To increase the probability of finding jumps it is necessary to have a priori information.
\end{abstract}

Keywords: A posteriori density probability; economic processes; jump detection; profitability parameters.

\section{Introduction}

Estimation of economic processes parameters of an enterprise, branch of economy or economy as a whole is an issue of current importance, especially during the crisis in Ukraine.

For other European countries, this is also relevant in connection with the growing volatility and problems of VUCA world (volatility, uncertainty, complexity and ambiguity). The functioning of any economic system in the modern VUCA world makes economic processes quick and unpredictable. A complex system can move from one state to another jump, that is within a very short period of time. The jumps of such economic parameters as profitability, economic value added, net present value, etc. could not be detected on time without the proper mathematical method using. In general, behaviour of an economic system could be described by stochastic process, as a sum of quasi-determined signal and noise which distorts real information about the process. Jumps are considered to be rapid or utterly rapid changes of stochastic processes parameters describing a system behaviour.

For appearance of jumps some reasons are necessary which could be either objective or subjective. The first type often occur in physical processes, and the second one, for example, in the economy. Different public phenomena, decision of government etc. could cause the jumps of economic parameters, both positive and negative. Knowledge of jumps parameters is an important factor for the management an economy. Basic parameters, as a rule, are an intensity of jump, its dispersion and the moment of its appearance. For the estimation of aforementioned parameters proper stochastic models are created.

\section{Review of Literature}

At the basis of each model a stochastic process lies. It was first studied in statistical physics. The simplest models of a random process are the Wiener process that describes the Brownian motion of particles of matter, and the Poisson process. These two processes are a special case of the Lévy process used in [1] to evaluate the switching mode. Lévy process is a stochastic process with independent stationary increments and describes the movement of a point, whose successful movements are random and statistically independent at intervals of the same duration. The evaluation of the strategy in [1] is based on a two-step procedure for evaluating the diffusion of parameters in the presence of switching and estimating the Lévy jump component by means of inverse Gaussian distributions adapted to each mode. Gaussian and Markov random processes are widely used in practice. There are no jumps in all of these random process models. That is why in practice models of jumps are either intentionally introduced into the model of a random process, or a jump is considered to be an abnormal value of the process parameter. For this, of course, some threshold is used and the excess of threshold being treated as a jump. For example, in [2] the threshold sets for the test statistics of the Dow Jones index.

Random processes are often modulated in the form of the sum of a deterministic process, which is subject to some laws, and noise. In [3], noise is a market-based microstructural noise, which makes it difficult to detect a jump in asset prices. In [4], the model has a market and an individual component. In [5], the financial series are described conventionally by the Gaussian model that is "contaminated" by jump components. Noise in models is considered to be white or even multiplicative white noise [6]. To 
describe the economic processes of updating (renewal process), [7] uses the continuous random walk process (CTRW), which was previously introduced in physics as a model of standard and abnormal diffusion. CTRW processes can be both Markov and semi Markov. To simulate such processes, the Monte Carlo method is widely used. Many models of jump-diffusion processes [8] have been proposed to describe asset prices.

The basis of the approach to detecting jumps of the parameters of economic processes are different statistical tests. In [3], the researched local average is used for short jumps, and in [9] there are a number of tests: CPR, Corsi, Pirino, Reno, MEDR, each of which has its own efficiency for different conditions. Both parametric and nonparametric tests are considered, in particular [10] uses the asymptotic distribution for nonparametric tests. In [11], based on the use of intraday highs and minima of asset prices, the conditions for detecting jumps, exchange rates, etc. are investigated. With the help of jump-diffusion processes [8], the $\mathrm{CRB}$ index is evaluated for its further use in assessing systematic price risks. Models of Stratonovich and Marcus [6] are constructed so that, for appropriate conditions, it would be possible to operate with stochastic integrals as with ordinary ones. In [6], Marcus canonical equation is introduced as the limiting equation of systems described by the model of the Levy-OrnsteinUhlenbeck using colour noise. In order to detect jumps in financial markets, the use of bi-power variations, which makes it possible to obtain the intensity of jumps, the mean value and the variance is proposed in [12, 13]. In [14], a test is proposed to determine the jump in the return of assets or other discrete processes. The test does not depend either on the law of the process or on the coefficients of the solvable differential equations, does not require a preliminary value of these coefficients and does not depend on the activity of the jumps.

The main drawbacks of the methods described above for detecting jumps are:

- all methods are based on the use of statistical tests that are not universal and are well suited for analyzing only individual economic processes;

- the results of detecting jumps of process parameters are too inertial and appear after processing of a large amount of statistical material;

- most methods do not require a priori knowledge of the stochastic process, but really such knowledge exists;

- the proposed methods do not provide clear settings for the intensity of noise, in which it is possible to detect the jump; - many of these methods do not simultaneously provide information on the intensity of the detected jump, the accuracy of its definition;

- in the works, the probability of finding a jump for specific conditions is not evaluated.

Almost all of these shortcomings are eliminated by the method proposed in the article. It is based on the Fokker-PlanckKolmogorov equation in the form of a system of differential equations in partial derivatives, which was initially obtained in statistical mechanics. It describes the evolution in time of a posteriori probability density of the investigated parameter of a process. On the basis of this equation, Stratonovich received an equation that is most suitable for describing rapid radio engineering processes. It tracks the maximum of a posteriori probability density of the investigated parameter.

\section{Objective of the Study}

The objective of the study is to develop a method for detecting and estimating the jumps of the economic processes parameters, for example, the profitability of a separate economic system.

\section{System of Detection and Measurement of Rapid Changes in the Parameters of Profitability}

Let's develop a system for detecting and evaluating the jumps of profitability parameters, considering the profitability as a continuous random process. To obtain a system of differential equations we use the Stratonovich equation [15] and the method of averaging over time [16]. Consequently, the random process of profitability is observed by some system (receiver) that is able to estimate profitability and its components. However, it is difficult to obtain the exact value of profitability, since there is a large number of unpredictable factors that interfere with the work of the receiver and create noise. In some cases, there is a steep increase or decrease in profitability, that is, a jump in the profitability parameter or overall profitability. This jump may be omitted due to the presence of noise. Hence the problem of detecting jumps of these parameters and estimating their level in conditions of interference or noise arises.

We have built a model of profitability of operational activity of enterprises of Kharkiv region by types of industrial activity (machine building) based on data for 2010 - 2016 [17]; the exponential smoothing method was used in Excel.

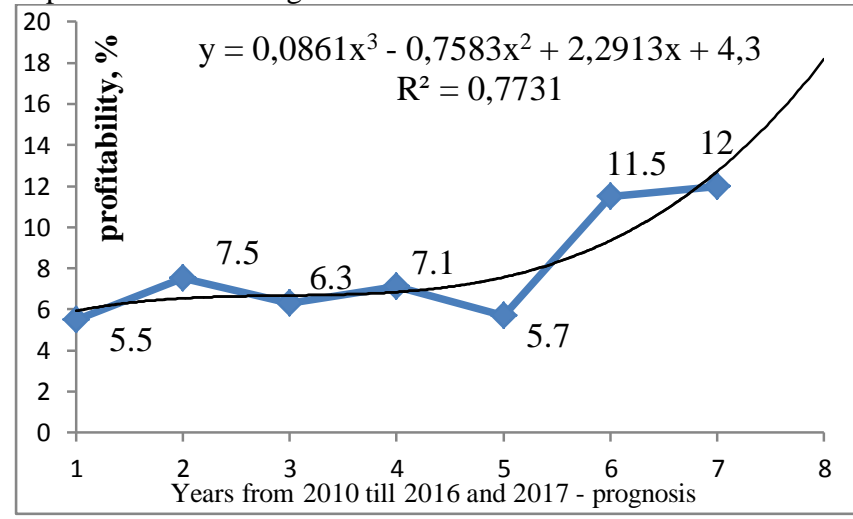

Fig. 1: Graph of actual and simulated values of profitability of operational activity of Kharkiv region enterprises by types of industrial activity (machine building).

The model is described by the polynomial of the third degree

$r(t)=\eta_{0}+\eta_{1} t+\eta_{2} t^{2}+\eta_{3} t^{3}=\sum_{i=0}^{3} \eta_{i} t^{i}$

where $t$ - the time, $\eta_{i}$ - the dimensional coefficients, which, for given conditions are:

$\eta_{0}=4.3 ; \eta_{1}=2.2913 ; \eta_{2}=-0.7583 ; \eta_{3}=0.861$.

The graph of the function $r(t)$ (Fig. 1) in order to simplify the further presentation can be approximated by a quadratic function, which is determined by the method of least squares

$$
r_{a p}(t)=4.3+0.27 t^{2}
$$

Taking into consideration noise at the input of the receiver (the register of profitability) there will be an occasional process

$y(t)=r(\vec{\eta}, t)+n(t)$

where $r(\vec{\eta}, t)$ - the expected function of profitability, which we will consider quadratic, $n(t)$ - white Gaussian noise, with zero mean value and spectral intensity; $\vec{\eta}=\left\{\eta_{i}\right\}_{i=0}^{i=3}$ - a vector of dimensional coefficients, which describes the function of profitability. In the subsequent study, the vector $\vec{\eta}$ will have two 
or one unknown in the general case of a component that is statistically independent of noise $n(t)$.

There is a possibility of a jump-like change of profitability which is mathematically described as

$$
y(t)=\left\{\begin{array}{l}
\alpha\left(\beta_{0}+\beta t^{2}\right)+n(t) \text { when } t \leq \tau, \\
(\alpha+\Delta \alpha)\left(\beta_{0}+(\beta+\Delta \beta) t^{2}\right)+n(t) \text { when } t>\tau .
\end{array}\right.
$$

where $\alpha$ - the dimensionless coefficient, for example, $\alpha=1$; $\Delta \alpha$ - a jump of dimensionless coefficient which characterizes the jump in profitability. On the other hand, after a profitability jump the function type $r(\vec{\eta}, t)$ may change significantly. Such a change is described by the coefficient jump in the function of profitability, that is, the unknown value $\Delta \beta$ shown in (3). Note that the jump of the economic processes parameters must be understood as a rapid (abrupt) change in the parameter in a time that does not exceed the period of registration of this process, in our case, profitability. Suppose further that the random jump moment $\tau_{\text {jump }}$ does not depend on noise $n(t)$ and is a priori distributed by the Gaussian law with mean values $\bar{\tau}_{\text {jump }}$ and variances $\sigma_{\tau_{\text {jump }}}^{2}$.

In the problem we are interested in jumping two parameters that characterize profitability: $\Delta \alpha$ and $\Delta \beta$. They are components of a random vector process $\vec{x}(t)$. The priori equation describing the probability density of this vector process has the form

$$
\frac{\partial W_{x}(\vec{x}, t)}{\partial t}=L W_{x}(\vec{x}, t),
$$

where $L$ is the Fokker-Planck operator which takes different expressions before and after the jump, i. e.

$$
L(\square)=\left\{\begin{array}{l}
L_{0}(\square) \text { when } t \leq \tau_{\text {jump }}, \\
L_{1}(\square) \text { when } t>\tau_{\text {jump }} .
\end{array}\right.
$$

In practice, the magnitude of jumps $\Delta \alpha, \Delta \beta$ at the jump moment $\tau_{\text {jump }}$ is usually a priori unknown. The problem consists in the fact that, on the realization $\mathrm{y}(\mathrm{t})$ of a random process that describes the profitability, on the interval $(0, t)$ to obtain the optimal in the mean square estimation $\vec{x}(t)$ of the of the state vector $\vec{x}(t)$ filtering. For a complete definition of this problem, it is necessary at the initial time $t=0$ to set a compatible a priori probability density $P_{x \tau}(\vec{x}, \tau)$ of random totality $\{\vec{x}, \tau\}$.

Using the general results of the theory of random Markov processes for the a posterior density of the probability of the Markov totality $\{\vec{x}, \tau\}$ let's write the Stratonovich equation [16]

$$
\frac{\partial W_{x \tau}(\vec{x}, \tau ; t)}{\partial t}=L W_{x \tau}(\vec{x}, \tau ; t)+\left[F(\vec{x}, \tau, t)-<F(\vec{x}, \tau, t)>_{x \tau}\right] \cdot W_{x \tau}(\vec{x}, \tau ; t),
$$

with the initial condition

$$
t \geq 0
$$

$$
\left.W_{x \tau}(\vec{x}, \tau ; t)\right|_{t=0}=P_{x \tau}(\vec{x}, \tau) .
$$

Here $L(\square)$ - the same operator as in the equation (4), and the function $F(\vec{x}, \tau, t)$ is determined by the expression [16]

$$
F_{i}(\vec{x}, \tau, t)=S_{i}^{T}(\vec{x}, t) N^{-1}\left[y(t)-\frac{1}{2} S_{i}(\vec{x}, t)\right]=\left\{\begin{array}{l}
F_{0}(\vec{x}, t) \text { when } t \leq \tau, \\
F_{1}(\vec{x}, t) \text { when } t>\tau, i=1,2 .
\end{array}\right.
$$

$<F(\vec{x}, \tau, t)>_{x \tau}=\iiint_{-\infty}^{\infty} F(\vec{x}, \tau, t) W_{x \tau}(\vec{x}, \tau ; t) d \vec{x} d \tau$,

where $N^{-1}$ - the matrix which is the inverse matrix of the spectral intensity of the observations noise in the model, and the other functions are determined by the formulas:

$$
\begin{aligned}
& S_{0}(\Delta \alpha, \Delta \beta, t)=\alpha\left(\beta_{0}+\beta t^{2}\right)=S_{0}(t) \\
& S_{1}(\Delta \alpha, \Delta \beta, t)=(\alpha+\Delta \alpha)\left[\beta_{0}+(\beta+\Delta \beta) t^{2}\right], \\
& F_{0}(t)=\frac{2 y(t) S_{0}(t)-S_{0}^{2}(t)}{N_{0}}, \\
& F_{1}(\Delta \alpha, \Delta \beta, t)=\frac{2 y(t) S_{1}(\Delta \alpha, \Delta \beta, t)-S_{1}^{2}(\Delta \alpha, \Delta \beta, t)}{N_{0}}
\end{aligned}
$$

By introducing some additional functions we transform (6). Let $W_{x}(\vec{x}, t)$ and $W_{\tau}(\tau, t)$ - one-dimensional a posteriori density probabilities of the vector $\vec{x}$ and moment of a jump $\tau$ at time t:

$$
\begin{aligned}
& W_{x}(\vec{x}, t)=\int_{-\infty}^{\infty} W_{x \tau}(\vec{x}, \tau ; t) d t \\
& W_{\tau}(\tau, t)=\int_{-\infty}^{\infty} W_{x \tau}(\vec{x}, \tau ; t) d \vec{x}
\end{aligned}
$$

We introduce a posteriori probabilities of occurrence and absence of a jump of the profitability parameter by the time moment $t$

$$
\begin{aligned}
& p_{1}(t) \mid=\int_{-\infty}^{t} W_{\tau}(\tau ; t) d \tau, \\
& p_{0}(t) \mid=\int_{t}^{\infty} W_{\tau}(\tau ; t) d \tau,
\end{aligned}
$$

and probability density $W_{0}(\vec{x}, t)$ and $W_{1}(\vec{x}, t)$ by means of relationships

$$
\begin{aligned}
& W_{0}(\vec{x}, t)=\frac{1}{p_{0}(t)} \int_{t}^{\infty} W_{x \tau}(\vec{x}, \tau ; t) d \tau, \\
& W_{1}(\vec{x}, t)=\frac{1}{p_{1}(t)} \int_{-\infty}^{t} W_{x \tau}(\vec{x}, \tau ; t) d \tau .
\end{aligned}
$$

The derivation of a system of stochastic differential equations (SDE) for determining the jumps of random process parameters is given in the appendix using materials $[18,19]$.

Let's consider the system of optimal measuring the magnitude of the jumps of profitability parameters in an unknown a priori moment of time $\tau$. We assume that the signal shows the change of parameters and has the form (3). Here, the parameters $\alpha, \beta_{0}, \beta$ and white Gaussian noise $n(t)$ with spectral intensity $0.5 \cdot N_{0}$, which is easy to simulate on a computer, are known. Unknown values in (3) are profitability jumps that arise in an unknown a priori moment of time $\tau$ and are described by parameters $\Delta \alpha, \Delta \beta$. There are theoretically possible situations of a jump $\Delta \alpha$ occurrence without a jump $\Delta \beta$ at a given time moment $\tau$ or a jump $\Delta \beta$ without a jump $\Delta \alpha$. The most probable situation is the appearance of both jumps, that is, $\Delta \alpha$ and $\Delta \beta$. We assume that at the initial moment of time $t=0$ a priori probability density $P_{\tau}(\tau), P_{\Delta \alpha}(\Delta \alpha)$ and $P_{\Delta \beta}(\Delta \beta)$ are given. An apriori compatible distribution of the probability density of the specified parameters or two of them can also be specified. Everything is determined by the statement of the problem. 
Consequently, in the accepted notation we have a random vector to be evaluated $\vec{x}(t)=\left(\begin{array}{l}\Delta \alpha \\ \Delta \beta\end{array}\right)$ but the time of the jump occurrence $\tau$ and the jumps magnitudes $\Delta \alpha, \Delta \beta$ themselves are unknown. Assuming that they do not change with time, we obtain $L_{0}(\square)=L_{1}(\square)=0$. Substituting the relation (9) to (12) into equation (6), we get

$\frac{d p_{1}}{d t}=P_{\tau}(t) \exp ^{-z}+p_{1}\left(1-p_{1}\right)\left[<F_{1}(\Delta \alpha, \Delta \beta, t)>_{1}-F_{0}(t)\right]$,

$\frac{d z}{d t}=p_{1}\left[<F_{1}(\Delta \alpha, \Delta \beta, t)>_{1}-F_{0}(t)\right]$,

$W_{0}(\Delta \alpha, \Delta A, t)=P_{\Delta \alpha \Delta \beta}(\Delta \alpha, \Delta \beta)$,

$\frac{\partial W_{1}(\Delta \alpha, \Delta \beta, t)}{\partial t}=\frac{P_{\tau}(t) \exp ^{-z}}{p_{1}}\left[P_{\Delta \alpha \Delta \beta}(\Delta \alpha, \Delta \beta)-W_{1}(\Delta \alpha, \Delta \beta, t)\right]+$
$+\left[F_{1}(\Delta \alpha, \Delta \beta, t)-<F_{1}(\Delta \alpha, \Delta \beta, t)>_{1}\right] W_{1}(\Delta \alpha, \Delta \beta, t)$

We will use the Gaussian approximation of functions $W_{0}(\Delta \alpha, \Delta \beta, t), W_{1}(\Delta \alpha, \Delta \beta, t)$.

$$
\begin{gathered}
W_{0,1}(\Delta \alpha, \Delta \beta, t)=\frac{1}{2 \pi \sigma_{\Delta \alpha} \sigma_{\Delta \beta} \sqrt{1-\rho^{2}}} \exp \left\{-\frac{1}{2\left(1-\rho^{2}\right)}\left[\frac{(\Delta \alpha-\overline{\Delta \alpha})^{2}}{\sigma_{\Delta \alpha}{ }^{2}}-\right.\right. \\
\left.\left.-2 \rho \frac{(\Delta \alpha-\overline{\Delta \alpha})(\Delta \beta-\Delta \bar{\beta})}{\sigma_{\Delta \alpha} \sigma_{\Delta \beta}}+\frac{(\Delta \beta-\Delta \bar{\beta})^{2}}{\sigma_{\Delta \beta}{ }^{2}}\right]\right\},
\end{gathered}
$$

where $\rho$ - the correlation coefficient between jumps $\Delta \alpha$ and $\Delta \beta, \sigma_{\Delta \alpha}^{2}, \sigma_{\Delta \beta}^{2}$ - the variances of the magnitude fluctuations of the corresponding jumps $\Delta \alpha$ and $\Delta \beta, \overline{\Delta \alpha}, \overline{\Delta \beta}$ - are their mean values.

In the ratio (20) there are no indexes for the corresponding parameters. The function $W_{0}(\Delta \alpha, \Delta \beta, t)$ characterizes the a priori probability density of the profitability parameters before jumps, and the function $W_{1}(\Delta \alpha, \Delta \beta, t)$ - after the jumps. The average function in the equations $(16,17,19)$ is calculated as follows:

$<F_{1}(\Delta \alpha, \Delta \beta, t)>_{1}=\int_{0}^{\Delta \beta_{m}}\left(\int_{0}^{\Delta \alpha_{m}} F_{1}(\Delta \alpha, \Delta \beta, t) W_{0,1}(\Delta \alpha, \Delta \beta, t) d \Delta \alpha\right) d \Delta \beta$,

where $\Delta \alpha_{m}, \Delta \beta_{m}$ - the maximum expected magnitudes of jumps $\Delta \alpha$ and $\Delta \beta$.

In the system of SDE (16) ... (19) almost all the necessary functions are already defined, except for the a priori distribution of time jump occurrence $P_{\tau}(t)$, which we also consider to be distributed according to the Gaussian law with the expected mean value of the jump time $t_{\tau}$ and the variance of this time $\sigma_{\tau}{ }^{2}$. The function $P_{\Delta \alpha \Delta \beta}(\Delta \alpha, \Delta \beta)$ in the ratio (18) describes the a priori distribution of the jump parameters $\Delta \alpha, \Delta \beta$.

Let's consider the differential equation of the system (19). It, with the help of other equations of the system, allows us to estimate the maximum of a posteriori density of the probability of profitability $W_{1}(\Delta \alpha, \Delta \beta, t)$, i. e. in the ideal case, the right part of this equation should be close to zero. Determination of the global minimum of the right-hand side (19) as a function of two variables $\Delta \alpha, \Delta \beta$ was carried out in the process of solving the system of equations by stochastic search using a genetic algorithm.

To evaluate the system workability for detecting profitability jumps, let us give an example of a rapid parameters change (Fig.
2) which are part of the system of differential equations (16) ... (19). Due to the such behaviour of these parameters, the profitability changes by a jump (Fig. 3, a) at some time moment and under noise conditions this dependence takes the form, as shown in Fig. 3, b or Fig. 3, c.
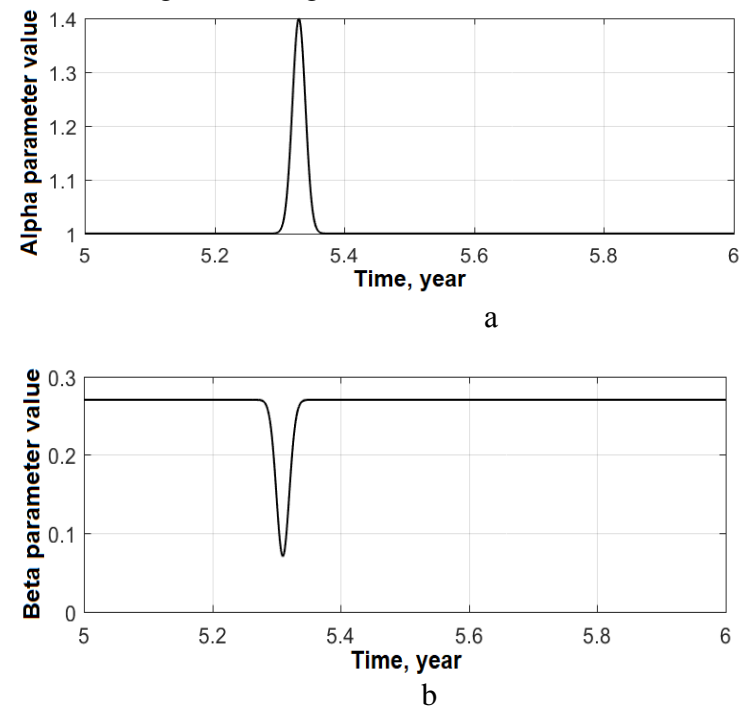

Fig. 2: The examples of parameters jumps $\alpha$ (a) and $\beta$ (b)

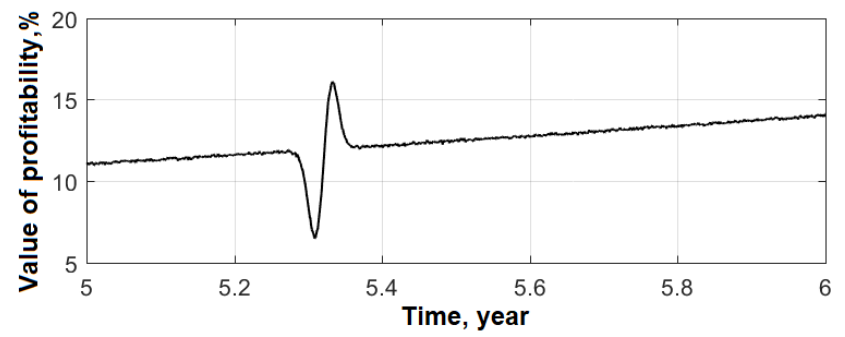

a

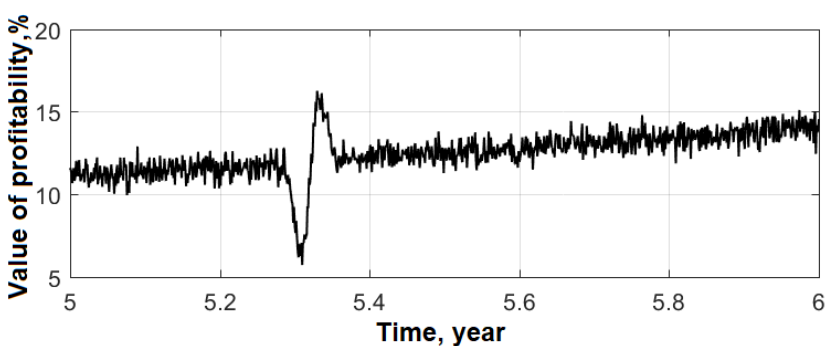

b

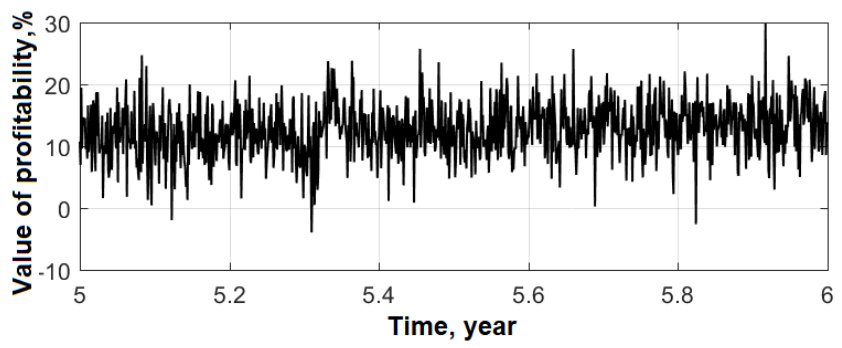

c

Fig. 3: The examples of profitability jumps during the year in the absence (a) and presence of noise (b, c).

In Fig. 3, b the ratio of the jump amplitude to the amplitude of noise is ten, and in Fig. 3, $\mathrm{c}$ is a unit.

Fig. 4 shows the time dependence of the jump probability detection for conditions that correspond to the graph in Fig. 3, c. 


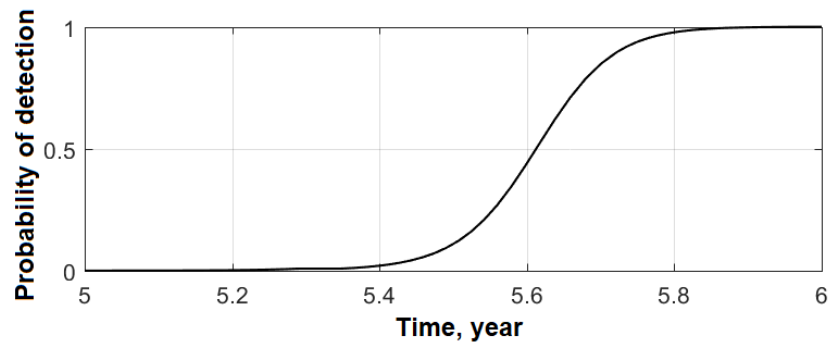

Fig. 4: Dependence of the probability of jump profitability detection from time for conditions presented in Fig. 3, c .

At the jump moment this probability is close to zero, but over time, it increases and reaches a value close to one, that is, the jump profitability detection system turns out to be a delayed system. This is confirmed by the speed of the system's operation, which is determined by equation (17) (Fig. 5).

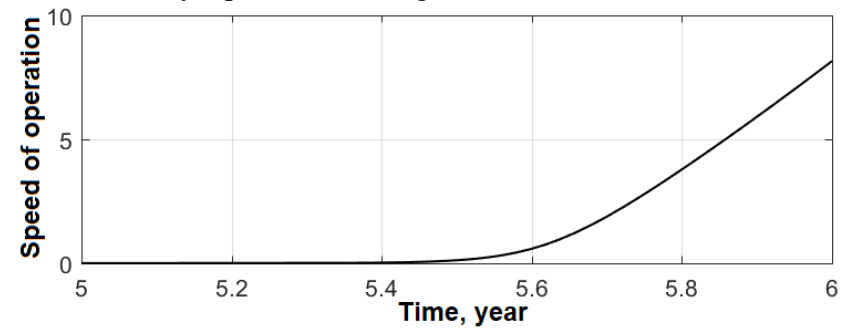

Fig. 5: Speed of operation of the jump profitability detection system in relative units.

As it follows from Fig. 5, the speed $z$ of the detection system at the time of jump is zero, and after some time it increases, which makes it possible to detect a profitability jump.

As already noted, the solution of the SDE (16) to (19) is accompanied by the search for a maximum of a posteriori probability density $W_{1}(\Delta \alpha, \Delta \beta, t)$ and is not attractive and convenient for obtaining practical results. We synthesize a system of stochastic differential equations for a case where only one parameter $\Delta \alpha$ determines the jump of profitability. The signal model for processing in the system is similar (3), but in it $\Delta \beta=0$. We assume that at the initial moment of time given a priori probabilities density $P_{\tau}(\tau)$ and $P_{\Delta \alpha}(\Delta \alpha)$ are given. The prior compatible distribution of the probability density of the specified parameters or two of them may also be known. Therefore, in the accepted notation, the random vector $\vec{x}(t)$ to be evaluated has only one component $\Delta \alpha$, but the moment of the jump occurrence and the value of the jump itself $\Delta \alpha$ are unknown. All the necessary functions for compiling the system of differential equations and describing the jump $\Delta \alpha$ are determined in the same way as the relations (9) to (12). Using the functions $W_{0}(\Delta \alpha, t), W_{1}(\Delta \alpha, t)$ we obtain the system of equations which is analogous to the system (16) to (19):

$$
\frac{d p_{1}}{d t}=P_{\tau}(t) \exp ^{-z}+p_{1}\left(1-p_{1}\right)\left[<F_{1}(\Delta \alpha, t)>_{1}-F_{0}(t)\right],
$$

$$
\frac{d z}{d t}=p_{1}\left[<F_{1}(\Delta \alpha, t)>_{1}-F_{0}(t)\right]
$$

$$
\begin{aligned}
& K_{\Delta \alpha}(t) \cdot \frac{d \Delta \alpha(t)}{d t}=\frac{P_{\tau}(t) \exp ^{-z}}{2 p_{1}}\left[P_{\Delta \alpha}(\Delta \alpha)-W_{1}(\Delta \alpha, t)\right]+ \\
& +F_{1}(\Delta \alpha, t) \cdot W_{1}(\Delta \alpha, t),
\end{aligned}
$$

$$
\begin{aligned}
& K_{d}(t) \cdot \frac{d D_{\Delta \alpha}(t)}{d t}= \\
& =\frac{P_{\tau}(t) \exp ^{-z}}{2 p_{1}}\left[P_{\Delta \alpha}(\Delta \alpha)-W_{1}(\Delta \alpha, t)\right]-<F_{1}(\Delta \alpha, t)>_{1} W_{1}(\Delta \alpha, t),
\end{aligned}
$$

where $K_{\Delta \alpha}(t)=\frac{\sqrt{2}}{2} \frac{E_{1}(t)}{D_{\Delta \alpha}^{3 / 2}(t) \sqrt{\pi}}(\overline{\Delta \alpha}-\Delta \alpha(t))$

$$
K_{d}(t)=\frac{\sqrt{2} E_{1}(t)}{4 \sqrt{\pi} D_{\Delta \alpha}^{3 / 2}(t)}\left(-1+\frac{\Delta \alpha^{2}(t)}{D_{\Delta \alpha}(t)}-\frac{2 \Delta \alpha \cdot \overline{\Delta \alpha}}{D_{\Delta \alpha}(t)}+\frac{\overline{\Delta \alpha}^{2}}{D_{\Delta \alpha}(t)}\right),
$$$$
E_{1}(t)=\exp \left(-\frac{1}{2 D_{\Delta \alpha}(t)}\left(\Delta \alpha^{2}(t)-2 \Delta \alpha(t) \overline{\Delta \alpha}+\overline{\Delta \alpha}^{2}\right)\right),
$$$$
E_{2}(t)=\exp \left(-\frac{\overline{\Delta \alpha}^{2}}{2 \sigma_{\Delta \alpha}^{2}(t)}\right),
$$$$
<F_{1}(\Delta \alpha, t)>_{1}=\bar{F}_{1}(t)=\frac{n(t)}{2 N_{0}}\left[\Phi_{1} n(t)+\Phi_{2} n(t)+2 \Phi_{1} \alpha \beta_{0}+2 \Phi_{1} \alpha \beta t^{2}+\right.
$$$$
\left.+2 \Phi_{2} \alpha \beta_{0}+2 \Phi_{2} \alpha \beta t^{2}\right]+\frac{1}{2 N_{0} \sqrt{\pi}}\left[\sqrt{\pi} \Phi_{1} \alpha^{2} \beta^{2} t^{4}-\sqrt{\pi} \Phi_{1} \beta^{2} \sigma_{\Delta \alpha}^{2} t^{4}-\right.
$$$$
-\sqrt{\pi} \Phi_{1} \beta^{2} \overline{\Delta \alpha}^{2} t^{4}+\Delta \alpha_{m} \sqrt{2} E_{1} \beta_{0}^{2} \sqrt{D_{\Delta \alpha}(t)}+\sqrt{2} E_{1} \beta_{0}^{2} \sqrt{D_{\Delta \alpha}(t)} \overline{\Delta \alpha}+
$$$$
+2 \sqrt{2} \Delta \alpha_{m} E_{1} \beta_{0} \beta \sqrt{D_{\Delta \alpha}(t)} t^{2}+2 \sqrt{2} E_{1} \beta_{0} \beta \sqrt{D_{\Delta \alpha}(t)} \overline{\Delta \alpha} t^{2}-
$$$$
-2 \sqrt{2} E_{2} \beta_{0} \beta \sqrt{D_{\Delta \alpha}(t)} \overline{\Delta \alpha}^{2} t^{2}+\sqrt{2} \Delta \alpha_{m} E_{1} \beta^{2} \sqrt{D_{\Delta \alpha}(t)} t^{4}+2 \sqrt{\pi} \Phi_{1} \beta_{0} \beta \alpha^{2} t^{2}-
$$$$
-2 \sqrt{\pi} \Phi_{1} \beta_{0} \beta D_{\Delta \alpha}(t) t^{2}-2 \sqrt{\pi} \Phi_{1} \beta_{0} \beta \overline{\Delta \alpha}^{2} t^{2}+\sqrt{2} E_{1} \beta^{2} \sqrt{D_{\Delta \alpha}(t)} \overline{\Delta \alpha} \cdot t^{4}-
$$$$
-\sqrt{2} E_{2} \beta^{2} \sqrt{D_{\Delta \alpha}(t)} \overline{\Delta \alpha} \cdot t^{4}+\sqrt{\pi} \Phi_{1} \beta_{0}^{2} \alpha^{2}-\sqrt{\pi} \Phi_{1} \beta_{0}^{2} D_{\Delta \alpha}(t)-\sqrt{\pi} \Phi_{1} \beta_{0}^{2} \overline{\Delta \alpha}^{2}+
$$$$
+\sqrt{\pi} \Phi_{2} \beta_{0}^{2} \alpha^{2}-\sqrt{\pi} \Phi_{2} \beta_{0}^{2} D_{\Delta \alpha}(t)-\sqrt{\pi} \Phi_{2} \beta_{0}^{2} \overline{\Delta \alpha}^{2}-\sqrt{2} E_{2} \beta_{0}^{2} \overline{\Delta \alpha}^{2} \sqrt{D_{\Delta \alpha}(t)}+
$$$$
+\sqrt{\pi} \Phi_{2} \alpha^{2} \beta^{2} t^{4}-\sqrt{\pi} \Phi_{2} \beta^{2} D_{\Delta \alpha}(t) t^{4}-\sqrt{\pi} \Phi_{2} \beta^{2} \overline{\Delta \alpha}^{2} t^{4}+2 \sqrt{\pi} \Phi_{2} \alpha^{2} \beta_{0} \beta t^{2}-
$$$$
\left.-2 \sqrt{\pi} \Phi_{2} \beta_{0} \beta D_{\Delta \alpha}(t) t^{2}-2 \sqrt{\pi} \Phi_{2} \beta_{0} \beta \overline{\Delta \alpha}^{2} t^{2}\right] \text {, }
$$

$\Phi_{1}=\operatorname{erf}\left(\frac{1}{\sqrt{2}} \frac{\left(\Delta \alpha_{m}-\overline{\Delta \alpha}\right)}{\sqrt{D_{\Delta \alpha}(t)}}\right), \quad \Phi_{2}=\operatorname{erf}\left(\frac{1}{\sqrt{2}} \frac{\overline{\Delta \alpha}}{\sqrt{D_{\Delta \alpha}(t)}}\right)$,

$D_{\Delta \alpha}=\sigma_{\Delta \alpha}^{2}(t)$ - fluctuations variance of the jump magnitude $\Delta \alpha$, and $\sigma_{p r}{ }^{2}$ is the a prior value of this variance which does not change over time in the selected system of equations (22)...(25); $\overline{\Delta \alpha}$ - its average value which change in time (in modeling, we consider it constant); $\overline{\Delta \alpha}_{p r}$ - the average a priori value of the jump which is always unchanged, $\Delta \alpha_{m}$ is the maximum of the jump expected value. The "erf" symbol indicates the integral of probabilities.

In addition, for solving SDE, the appropriate initial conditions are required, for example, $p_{1}(t=0)=p_{1}(0)=p_{1}^{0}$, where $p_{1}^{0}$ is the a priori probability of a jump that equals some a priori value.

The system of differential equations (22) ... (25) differs from system (16) ... (19) by the fact that in it explicitly we have the equation for estimating the profitability jump (24) and its variance (25). For a separate case, the change of profitability without noise and in the conditions of noise is depicted in Fig. 6.

For simulation, the parameter $\alpha$ jumps of different amplitudes were selected (Fig. 7).

It is obvious from Fig. 6, that it is very difficult to detect profitability jump visually because it is suppressed by noise. The solution of the SDE system (22) to (25) allows us to detect these jumps with a high probability, as it is shown in Fig. 8. 
As it can be seen from Fig. 8, with an increase of the jump amplitude the probability of its detection increases. The jumps with an amplitude of $40 \%$ are detected with a probability of approximately 0.75 for given conditions. The graphs that characterize the speed of system operation have a similar form.

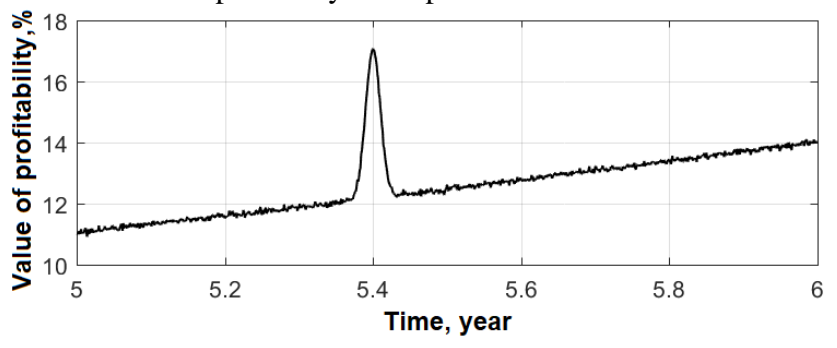

a

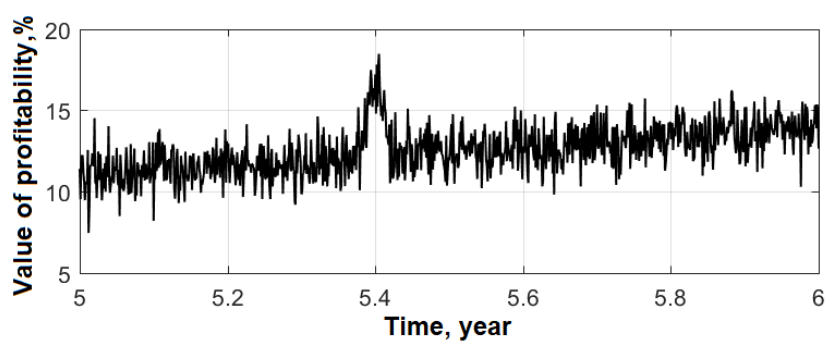

$\mathrm{b}$

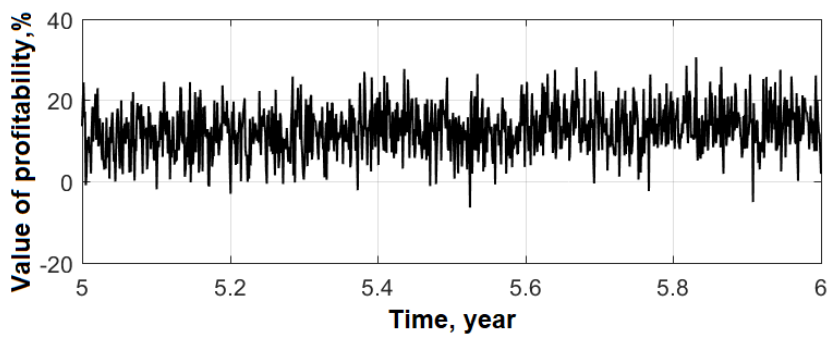

c

Fig. 6: Jump profitability during the year in noise conditions such as in Fig. 3 .

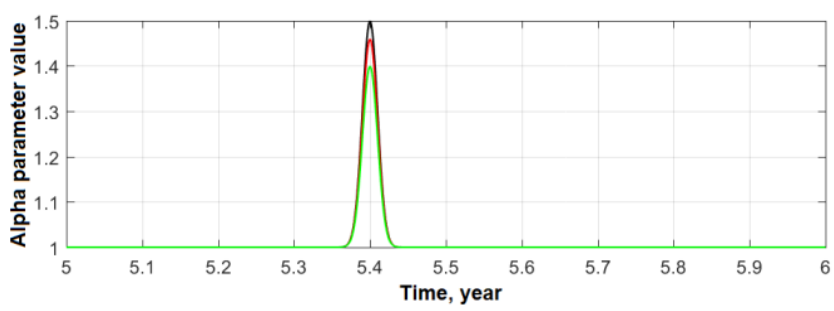

Fig. 7: Jump parameter $\alpha$ of three amplitudes: $40 \%, 45 \%$ and $50 \%$.

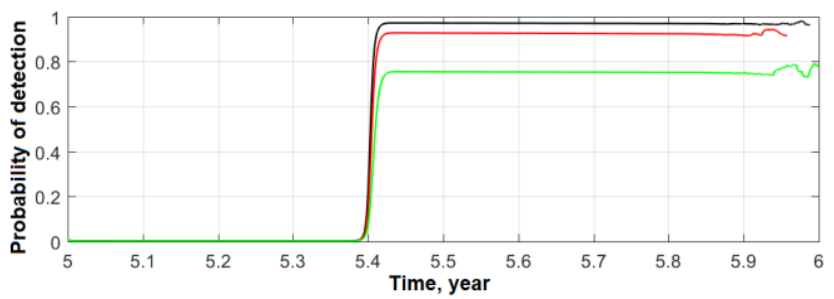

Fig. 8: Time dependence of the probability of the parameter $\alpha$ jumps detection for different jump amplitudes in noise conditions, such as in Fig. 6.

Note that the simulation of the system is carried out for a case where the noise components are practically identical with the magnitude of profitability, that is, in strict conditions and even in such conditions, the probability of jump profitability finding turns out to be quite high.

At the enterprises, and even in the industry as a whole, the daily profitability rate is not measured. According to statistical forms, at the enterprise, this happens periodically one time per month, quarter, year, and Region Administration will summarize annually. This fact makes the detection of jumps even more valuable for improving the management of economic objects. The method developed in the article allows to reveal both positive and negative advances of the economic index (in this case, profitability), and the diagnostic value of a negative jump detection is much higher because this information allows to identify the negative factors that reduce profitability and in the future can lead to a long-term decrease in profitability. But the identification of positive jumps is also valuable information that will enhance the impact of positive factors and make the temporary increase (jump) permanent or repeating itself.

\section{Conclusion}

A system of optimal detection and estimation of parameters jumps for economic processes on an example of profitability is synthesized in the article. The optimality of the system is ensured by finding the maximum of a posteriori probability density of jump profitability. The best situation for system operation is to detect and evaluate only one jump of the random process parameter. The main attention in the article is devoted to determining the probability of detecting jumps in different noise conditions. Even in the conditions of a high noise level which is commensurate with profitability, the high values (no less than 0.75 ) of probability of finding a jump are achieved. Detection of the jump is obtained with a delay relative to the moment of the jump and this is insignificant for economic processes. The probability of false jump detection is not considered in the article, although it can be obtained from the given formulas. Similarly, from the equations one can obtain both the numerical value of the jump and its variance. At enterprises the profitability rate is not measured daily. Hence, the role of the method of a profitability jump detection rises. The developed method can be used to detect the jumps of other parameters, for example, the exchange rate.

\section{References}

[1] Chevallier J., Goutte S., "Detecting jumps and regime switches in international stock markets returns", (2014), available online: https://hal.archives-ouvertes.fr/hal-01090833/document, last visit: 17.06.2018

[2] Sun Y., "Detecting Price Jumps in the Presence of Market Microstructure Noise", (2016), available online: https://pdfs.semanticscholar.org/ce07/04e8fb302802e895542a36fbe a92bc4585f8.pdf, last visit: 17.06.2018

[3] Bajgrowicz P. \& Scaillet O., Detecting spurious jumps in high frequency data, (2009), available online: http://www.ucd.ie/t4cms/scaillet-seminar-paper.pdf, last visit: 17.06.2018

[4] Maneesoonthorn W., Martin G.M. \& Forbes C.S., "Dynamic Asset Price Jumps and the Performance of High Frequency Tests and Measures", (2017), available online: https://www.monash.edu/business/econometrics-and-businessstatistics/research/publications/ebs/wp14-17.pdf, last visit: 17.06.2018

[5] Barndorff-Nielsen O.E. \& Shephard N., "Econometrics of testing for jumps in financial economics using bipower variation", Journal of Financial Econometrics, Vol.4, Issue 1, 1 January 2006, pp. 1-30, available online: https://doi.org/10.1093/jjfinec/nbi022, last visit: 17.06.2018

[6] Klößner S., "Grasping Economic Jumps by Sparse Sampling Using Intradaily Highs and Lows", (2011), available online: http://www.oekonometrie.unisaarland.de/papers/EconomicJumps.pdf, last visit: 17.06.2018

[7] Aravindhakshan S.C. \& Brorsen B.W. (2011), "Identifying Jumps and Systematic Risk in Futures." Proceedings of the NCCC-134 
Conference on Applied Commodity Price Analysis, Forecasting, and Market Risk Management. St. Louis, MO, available online: http://www.farmdoc.illinois.edu/nccc134/conf_2011/pdf/confp1311.pdf, last visit: 17.06 .2018

[8] Germano G., Politi M., Scadas E. \& Schilling R.L., "Itô and Stratonovich integrals on compound renewal processes: the normal/Poisson case", Commun Nonlinear Sci Numer Simulat, N15 (2010), pp. 1583-1588, available online: http://www.math.tudresden.de/sto/schilling/sources/pa/schilling33.pdf, last visit: 17.06.2018

[9] Chechkin A. \& Pavlyukevich I., "Marcus versus Stratonovich for Systems with Jump Noise", (2014), available online:

https://docopdf.com/marcus-versus-stratonovich-for-systems-with-jumpnoise.html, last visit: 17.06 .2018

[10] Tauchen G. \& Zhou H., "Realized Jumps on Financial Markets and Predicting Credit Spreads", (2006), available online: https://www.federalreserve.gov/pubs/feds/2006/200635/200635pap.p df, last visit: 17.06.2018

[11] Barndorff-Nielsen O.E., Power and bipower variation with stochastic volatility and jumps, (2003), available online:

https://pdfs.semanticscholar.org/7fd9/7ef4b68159046011513d924c5505a 039b924.pdf, last visit: 17.06.2018

[12] Ait-Sahalia Y. \& Jacod J., "Testing for Jumps in a Discretely Observed Process", (2009), available online:

https://arxiv.org/pdf/0903.0226.pdf, last visit: 17.06.2018

[13] Laurent S., Lecourt C. \& Palm F.C., "Testing for jumps in GARCH models, a robust approach", (2013), available online: https://cris.maastrichtuniversity.nl/portal/files/12733775/1744567.p df, last visit: 17.06 .2018

[14] Lee S.S. \& Wang M., "The Impact of Jumps on Carry Trade Returns," (2017), available online: https://pdfs.semanticscholar.org/a506/d37593377578e239c84ec174 7e6145d651c2.pdf, last visit: 17.06.2018

[15] Arxiv. Rentabel'nist operacijnoyi diyal nosti pidpry yemstv za vy'damy promy`slovoyi diyal'nosti, The site of the Main Directorate statistics in the Kharkiv region, available online: http://kh.ukrstat.gov.ua/index.php/rentabelnist-operatsiinoi-

diialnosti- pidpryiemstv-za-vydamy-promyslovoi-diialnosti, last visit: 14.06.2018

[16] Stratonovich R.L., Uslovnye markovskie processy i ih primenenie k teorii optimalnogo upravleniya, MGU. - Moscow, (1966), 319 p.

[17] Kay SM., Intuitive probability and random processes using MATLAB, University of Rhode Island Dept. of Electrical \& Computer Engineering.- Kingston, (2005), 835 p.

[18] Мальцев АА \& Силаев АМ. Оптимальное оченивание момента скачкообразного изменения статистических характеристик случайного процесса. - Изв. вузов. Радиофизика Mal’ev AA \& Sy`laev AM. Opty`mal’noe oceny`vany`e momenta skachkoobraznogo y`zmeneny`ya staty`sty`chesky`x xaraktery`sty`k sluchajnogo processa. - Y`zv. vuzov. Rady`ofy`zy`ka, 1986, №1, p.62-72.

[19] Polyarus OV, Barchan VV, Polyakov YeO \& Koval AO, (2009), “Opty`mal'na sy`stema vy`yavlennya i ocinyuvannya stry`bkiv amplitudy' vibracij dy`namichny'x ob'yektiv”, Xarkiv: East European Magazine of Advanced Technology, N6/6 (42), pp.21-23.

\section{Appendix - Derivation of the Stochastic Differential Equations System for the Parameters Jumps Estimation}

From formula (13) it is clear that

$$
W_{x}(\vec{x}, t)=p_{0}(t) W_{0}(\vec{x}, t)+p_{1}(t) W_{1}(\vec{x}, t)
$$

Using the relation (13), we find the equation for the introduced auxiliary functions $p_{1}(t), W_{0}(\vec{x}, t), W_{1}(\vec{x}, t)$. To do this, we differentiate the products $p_{0}(t) W_{0}(\vec{x}, t)$ and $p_{1}(t) W_{1}(\vec{x}, t)$ in time taking into account the representation (15) and equation (6).

$$
\begin{aligned}
& \frac{d W_{x}(\vec{x}, t)}{d t}=p_{0}(t) \frac{d W_{0}(\vec{x}, t)}{d t}+W_{0}(\vec{x}, t) \frac{d p_{0}(t)}{d t}+ \\
& p_{1}(t) \frac{d W_{1}(\vec{x}, t)}{d t}+W_{1}(\vec{x}, t) \frac{d p_{1}(t)}{d t} .
\end{aligned}
$$

$W_{x}(\vec{x}, t)=\int_{-\infty}^{t} W_{x \tau}(\vec{x}, \tau ; t) d \tau+\int_{t}^{\infty} W_{x \tau}(\vec{x}, \tau ; t) d \tau$

Hence,

$$
\begin{aligned}
& \frac{\partial W_{x}(\vec{x}, t)}{\partial t}=\int_{-\infty}^{\infty} \frac{\partial W_{x \tau}(\vec{x}, \tau ; t)}{\partial t} d \tau=\int_{-\infty}^{t} \frac{\partial W_{x \tau}(\vec{x}, \tau ; t)}{\partial t} d \tau+ \\
& +\int_{t}^{\infty} \frac{\partial W_{x \tau}(\vec{x}, \tau ; t)}{\partial t} d \tau .
\end{aligned}
$$

Derivatives under integrals are found by means of the relation (6), and we obtain

$$
\begin{aligned}
& p_{0}(t) \frac{\partial W_{0}(\vec{x}, t)}{\partial t}+W_{0}(\vec{x}, t) \frac{d p_{0}(t)}{d t}=-W_{x \tau}(\vec{x}, \tau ; t)+ \\
& +\int_{t}^{\infty}\left[L W_{x \tau}+\left(F-<F>_{x \tau}\right) W_{x \tau}\right] d \tau . \\
& p_{1}(t) \frac{\partial W_{1}(\vec{x}, t)}{\partial t}+W_{1}(\vec{x}, t) \frac{d p_{1}(t)}{d t}=-W_{x \tau}(\vec{x}, \tau ; t)+ \\
& +\int_{-\infty}^{t}\left[L W_{x \tau}+\left(F-<F>_{x \tau}\right) W_{x \tau}\right] d \tau .
\end{aligned}
$$

We integrate these expressions by $\vec{x}$ and apply (5), (8) and (14). As a result, an equation for probability $p_{1}(t)$ is obtained

$$
\begin{aligned}
& p_{1}(t) \int_{-\infty}^{\infty} \frac{\partial W_{1}(\vec{x}, t)}{\partial t} d \vec{x}+\frac{d p_{1}(t)}{d t} \int_{-\infty}^{\infty} W_{1}(\vec{x}, t) d \vec{x}= \\
& =\int_{-\infty}^{\infty} W_{x \tau}(\vec{x}, \tau ; t) d \vec{x}+\int_{-\infty}^{\infty} \int_{-\infty}^{t} \frac{W_{x \tau}(\vec{x}, t)}{\partial t} d \tau d \vec{x}+ \\
& \quad+\int_{-\infty}^{\infty} \int_{-\infty}^{t}\left(F_{1}-<F_{1}>_{x \tau}\right) W_{x \tau}(\vec{x}, \tau ; t) d \tau d \vec{x},
\end{aligned}
$$

$$
\begin{gathered}
p_{0}(t) \int_{-\infty}^{\infty} \frac{\partial W_{0}(\vec{x}, t)}{\partial t} d \vec{x}+\frac{d p_{0}(t)}{d t} \int_{-\infty}^{\infty} W_{0}(\vec{x}, t) d \vec{x}= \\
=-\int_{-\infty}^{\infty} W_{x \tau}(\vec{x}, \tau ; t) d \vec{x}+\int_{-\infty}^{\infty} \int_{t}^{\infty} \frac{W_{x \tau}(\vec{x}, t)}{\partial t} d \tau d \vec{x}+ \\
\quad+\int_{-\infty}^{\infty} \int_{t}^{\infty}\left(F_{0}-<F_{0}>_{x \tau}\right) W_{x \tau}(\vec{x}, \tau ; t) d \tau d \vec{x} .
\end{gathered}
$$

Let's denote

$$
\begin{aligned}
& \int_{-\infty}^{\infty} F_{1}(\vec{x}, t) W_{1}(\vec{x}, t) d \vec{x}=<F_{1}(\vec{x}, t)>_{1}, \\
& \int_{-\infty}^{\infty} F_{0}(\vec{x}, t) W_{0}(\vec{x}, t) d \vec{x}=<F_{0}(\vec{x}, t)>_{0} .
\end{aligned}
$$

$\frac{d p_{1}(t)}{d t}=W_{\tau}(\tau, t)+p_{1}\left(1-p_{1}\right)\left[<F_{1}(\vec{x}, t)>_{1}-<F_{0}(\vec{x}, t)>_{0}\right]$

$<F_{1}(\vec{x}, t)>_{1}=\int_{-\infty}^{\infty} F_{1}(\vec{x}, t) W_{1}(\vec{x}, t) d \vec{x}$,

$<F_{0}(\vec{x}, t)>_{0}=\int_{-\infty}^{\infty} F_{0}(\vec{x}, t) W_{0}(\vec{x}, t) d \vec{x}$ 
Let us represent the probability density $W_{x \tau}(\vec{x}, \tau ; t)$ in the form of the product of one-dimensional $W_{\tau}(\tau, t)$ and conditional $W_{x / \tau}(\vec{x} / \tau ; t) d \vec{x}$ densities

$W_{x \tau}(\vec{x}, \tau ; t) d \vec{x}=W_{\tau}(\tau, t) \cdot W_{x / \tau}(\vec{x} / \tau ; t) d \vec{x}$

Substituting (A8) and (A9) into (A4), we find the equation for the functions $W_{0}(\vec{x}, t)$ and $W_{1}(\vec{x}, t)$

$$
\begin{aligned}
& \frac{\partial W_{0}(\vec{x}, t)}{\partial t}=-\frac{W_{\tau}(\tau, t)\left[W_{x / \tau}(\vec{x} / \tau ; t) d \vec{x}-W_{0}(\vec{x}, t)\right]}{1-p_{1}}+ \\
& +L_{0} W_{0}(\vec{x}, t)+\left(F_{0}-<F_{0}>_{0}\right) W_{0}(\vec{x}, t) \\
& \frac{\partial W_{1}(\vec{x}, t)}{\partial t}=-\frac{W_{\tau}(\tau, t)\left[W_{x / \tau}(\vec{x} / \tau ; t) d \vec{x}-W_{1}(\vec{x}, t)\right]}{p_{1}}+ \\
& +L_{1} W_{1}(\vec{x}, t)+\left(F_{1}-<F_{1}>_{1}\right) W_{1}(\vec{x}, t)
\end{aligned}
$$

The initial conditions for the system of equations (A8) and (A10) are obtained from (7), (14), (15):

$\left.p_{1}(t)\right|_{t=0}=\int_{-\infty}^{0} P_{\tau}(\tau) d \tau=P_{10}$

$\left.W_{0}(\vec{x}, t)\right|_{t=0}=\frac{1}{1-P_{10}} \int_{0}^{\infty} P_{x \tau}(\vec{x}, t) d \tau$,

$\left.W_{1}(\vec{x}, t)\right|_{t=0}=\frac{1}{P_{10}} \int_{-\infty}^{0} P_{x \tau}(\vec{x}, t) d \tau$

Here $P_{\tau}(\tau) \equiv \int_{-\infty}^{\infty} P_{x \tau}(\vec{x}, t) d \vec{x}$ is the a priori probability density of the jump time.

The equations (A10) for functions $W_{0}(\vec{x}, t)$ and $W_{1}(\vec{x}, t)$ have the form similar to the Stratonovich equation for aposterior probability density. However, the right part of the equations (A10) includes additional components that determine the possibility of estimating a jump occurrence at the time $t$.

To ensure the system of equations (A8), (A10) is closed, it is necessary to determine a posteriori density of the probability $W_{\tau}(\tau, t)$ of the jump occurrence at the moment $t=\tau$ and the conditional probability density $W_{x / \tau}(\vec{x} / \tau ; t)$ of the distribution $\vec{x}$ at that time moment. Let's find the formula for $W_{\tau}(\tau, t)$ and $W_{x / \tau}(\vec{x} / \tau ; t)$ at arbitrary value of $\tau$. Integrating equation (6) by $\vec{x}$, we obtain the equation for the probability density $W_{\tau}(\tau, t)$ :

$\frac{\partial W_{\tau}(\tau, t)}{\partial t}=\left[G(\tau, t)-<G(\tau, t)>_{\tau}\right] W_{\tau}(\tau, t), \quad t \geq 0$,

$\left.W_{\tau}(\tau, t)\right|_{t=0}=P_{\tau}(\tau)$,

where

$G(\tau, t)=\int_{-\infty}^{\infty} F(\vec{x}, \tau, t) W_{x / \tau}(\vec{x} / \tau ; t) d \vec{x}=<F(\vec{x}, \tau, t)>_{x / \tau}$,

$<G(\tau, t)>_{\tau} \equiv \int_{-\infty}^{\infty} G(\tau, t) W_{\tau}(\tau, t) d \tau=<F(\vec{x}, \tau, t)>_{x / \tau}$

Substituting (A9) into (6) and using (A12), we find the equation for the conditional probability density $W_{x / \tau}(\vec{x} / \tau ; t)$ :

$$
\begin{gathered}
\frac{\partial W_{x / \tau}(\vec{x} / \tau ; t)}{\partial t}=L W_{x / \tau}(\vec{x} / \tau ; t)+ \\
+\left[F(\vec{x}, \tau, t)-<F(\vec{x}, \tau, t)>_{x / \tau}\right] W_{x / \tau}(\vec{x} / \tau ; t), \\
t \geq 0 .
\end{gathered}
$$

The initial condition for the equation (A13) is in (7):

$$
\left.W_{x / \tau}(\vec{x} / \tau ; t)\right|_{t=0}=P_{x / \tau}(\vec{x} / \tau)=P_{x \tau}(\vec{x}, \tau) / P_{\tau}(\tau)
$$

In the future we restrict ourselves to considering the case of independent initial distributions of the state vector $\vec{x}$ and the jump moment $\tau$. At the same time a posteriori values $\vec{x}$ and $\tau$ remain statistically independent. So,

$$
P_{x \tau}(\vec{x}, \tau)=P_{x}(\vec{x}) P_{\tau}(\tau)
$$

Then the initial condition for the equation (A13) will not depend on $\tau$ :

$\left.W_{x / \tau}(\vec{x} / \tau ; t)\right|_{t=0}=P_{x}(\vec{x})$

Let's consider the solution (A13) when $t \leq \tau$. Taking into account (5) and (8), the equation (A13) can be written as

$$
\begin{aligned}
& \frac{\partial W_{x / \tau}(\vec{x} / \tau ; t)}{\partial t}=L_{0} W_{x / \tau}(\vec{x} / \tau ; t)+ \\
& +\left[F_{0}(\vec{x}, t)-<F_{0}(\vec{x}, t)>_{x / \tau}\right] W_{x / \tau}(\vec{x} / \tau ; t),
\end{aligned}
$$

where $0 \leq t \leq \tau$ and the initial condition corresponds the condition (15). Since the operator $L_{0}(\square)$, function $F_{0}(\vec{x}, t)$ in (A17) and the initial condition (7) do not depend on $\tau$, then the solution of this equation also will not depend on $\tau$.

Let's compare the equation (A17) for the conditional probability density $W_{x / \tau}(\vec{x} / \tau ; t)$ with the first equation (A10) for the function $W_{0}(\vec{x}, t)$ and note that the initial conditions (A11) for the system (A8) and (A10), taking into account the a priori independence of the distributions $\vec{x}$ and $\tau$, take the form

$$
\begin{aligned}
& \left.W_{0,1}(\vec{x}, t)\right|_{t=0}=P_{x}(\vec{x}), \\
& \left.p_{1}(t)\right|_{t=0}=\int_{-\infty}^{0} P_{\tau}(\tau) d \tau .
\end{aligned}
$$

Therefore, the functions $W_{x / \tau}(\vec{x} / \tau ; t)$ and $W_{0}(\vec{x}, t)$ coincide at the initial moment of time $t_{0}=0$. Since the solution of the equation (A17) does not depend on $\tau$ at any $0 \leq t \leq \tau$, comparing the first equation of the system (A10) with (A17), we conclude that

$W_{x / \tau}(\vec{x} / \tau ; t) \equiv W_{0}(\vec{x}, t), 0 \leq t \leq \tau$

Thus, the desired conditional probability density $W_{x / \tau}(\vec{x} / \tau ; t)$ for all $t \geq 0$

$W_{x / \tau}(\vec{x} / \tau ; t) \equiv W_{0}(\vec{x}, t)$.

The equation for the auxiliary probability density $W_{0}(\vec{x}, t)$ taking into account (A20) acquires a simpler form

$$
\begin{aligned}
& \frac{\partial W_{0}(\vec{x}, t)}{\partial t}=L_{0} W_{0}(\vec{x}, t)+ \\
& +\left[F_{0}(\vec{x}, t)-<F_{0}(\vec{x}, t)>_{0}\right] W_{0}(\vec{x}, t), t \geq 0 .
\end{aligned}
$$

(A21)

The solution of the equation (A12) for $W_{\tau}(\tau, t)$ can be written as follows 
$W_{\tau}(\tau, t)=P_{\tau}(\tau) \exp \left\{\int_{0}^{t}\left[G\left(\tau, t^{\prime}\right)-<G\left(\tau, t^{\prime}\right)>_{\tau}\right] d t^{\prime}\right\}, t \geq 0$

(A22)

To calculate the value of a function $W_{\tau}(\tau, t)$ at a point $\tau=t$, it must be taken into account that the relations (A12), (A19) are performed when $t \geq t^{\prime}$

$G\left(\tau, t^{\prime}\right)=<F_{0}\left(\vec{x}, t^{\prime}\right)>_{x / \tau}=<F_{0}\left(\vec{x}, t^{\prime}\right)>_{0}$,

$<G\left(\tau, t^{\prime}\right)>_{\tau}=<F\left(\vec{x}, \tau, t^{\prime}\right)>_{x \tau}=P_{0}\left(t^{\prime}\right)<F_{0}\left(\vec{x}, t^{\prime}\right)>_{0}+$

$+P_{1}\left(t^{\prime}\right)<F_{1}\left(\vec{x}, t^{\prime}\right)>_{1}$

Substituting these expressions in (A22), we obtain

$W_{\tau}(\tau, t)=P_{\tau}(\tau) \exp ^{-z(t)}, t \geq 0$

$z(t)=p_{1}\left(t^{\prime}\right) \int_{0}^{t}\left[<F_{1}\left(\vec{x}, t^{\prime}\right)>_{1}-<F_{0}\left(\vec{x}, t^{\prime}\right)>_{0}\right] d t^{\prime}$.

The system of equations (A10), (A11) can now be written as:

$\frac{d p_{1}}{d t}=P_{\tau}(t) e^{-z(t)}+p_{1}\left(1-p_{1}\right)\left[<F_{1}\left(\vec{x}, t^{\prime}\right)>_{1}-<F_{0}\left(\vec{x}, t^{\prime}\right)>_{0}\right]$,

$\frac{d z}{d t}=p_{1}\left[<F_{1}\left(\vec{x}, t^{\prime}\right)>_{1}-<F_{0}\left(\vec{x}, t^{\prime}\right)>_{0}\right]$,

$\frac{\partial W_{0}(\vec{x}, t)}{\partial t}=L_{0} W_{0}(\vec{x}, t)+\left[F_{0}(\vec{x}, t)-<F_{0}(\vec{x}, t)>_{0}\right] W_{0}(\vec{x}, t)$,

$\frac{\partial W_{1}(\vec{x}, t)}{\partial t}=L_{1} W_{1}(\vec{x}, t)+\left[F_{1}(\vec{x}, t)-<F_{1}(\vec{x}, t)>_{1}\right] W_{1}(\vec{x}, t)+$

$+\left[\frac{P_{\tau}(t) e^{-z(t)}}{p_{1}}\right]\left[W_{0}(\vec{x}, t)-W_{1}(\vec{x}, t)\right]$,

$t \geq 0$

with initial conditions $\left.z(t)\right|_{t=0}=0$

The equations (A25) solve the problem of optimal nonlinear estimation of the state vector $\vec{x}$. Indeed, using the equation (A1), the optimal in the mean square sense the estimation $\vec{x}(t)$ of the state vector $\vec{x}(t)$ can be represented as the sum of two elementary estimates $\vec{x}_{0,1}(t)$, which are the mathematical expectations of the auxiliary probability planes $W_{0,1}(\vec{x}, t)$ :

$$
\begin{gathered}
\vec{x}_{0,1}(t)=\left[1-p_{1}(t)\right] \vec{x}_{0}(t)+p_{1}(t) \hat{\vec{x}}_{1}(t)= \\
=\left[1-p_{1}(t)\right] \int_{-\infty}^{\infty} \vec{x} \cdot W_{0}(\vec{x}, t) d \vec{x}+p_{1}(t) \int_{-\infty}^{\infty} \vec{x} \cdot W_{1}(\vec{x}, t) d \vec{x}
\end{gathered}
$$

From the solution of the equation (A25) we cannot find the optimal estimation $\hat{\tau}$ of the jump time $\tau$ at the time $t$, since from (A23), (A24) we will be aware of the values of the a posteriori probability density $W_{\tau}(\tau, t)$ only for $\tau=t$. However, using equation (14), it is easy to see that at the time $t_{0}$ when

$p_{1}\left(t_{0}\right)=1 / 2$,

the ratio

$\int_{-\infty}^{t_{0}} W_{\tau}\left(\tau, t_{0}\right) d \tau=\int_{t_{0}}^{\infty} W_{\tau}\left(\tau, t_{0}\right) d \tau$.

is executed

It follows directly that time $t_{0}$ is the optimal estimation of the jump time $\hat{\tau}_{\text {jump }}=t_{0}$

To clarify the estimation of the jump moment $\hat{\tau}_{\text {jump }}$, when $t>t_{0}$, it is necessary to solve a much more complicated equation (A12) for $W_{\tau}(\tau, t)$. 